\title{
Adaptive signal coloration maintained in the face of gene flow in a Hispaniolan Anolis Lizard
}

\author{
Julienne $\mathrm{Ng}^{1 *}$ D, Alison G. Ossip-Klein² and Richard E. Glor ${ }^{3}$
}

\begin{abstract}
Background: Studies of geographic variation can provide insight into the evolutionary processes involved in the early stages of biological diversification. In particular, multiple, replicated cases of geographic trait divergence present a powerful approach to study how patterns of introgression and adaptive divergence can vary with geographic space and time. In this study, we conduct replicated, fine-scaled molecular genetic analyses of striking geographic dewlap color variation of a Hispaniolan Anolis lizard, Anolis distichus, to investigate whether adaptive trait divergence is consistently associated with speciation, whereby genetic divergence is observed with neutral markers, or whether locally adapted traits are maintained in the face of continued gene flow.

Results: We find instances where shifts in adaptive dewlap coloration across short geographic distances are associated with reproductive isolation as well as maintained in the face of gene flow, suggesting the importance of both processes in maintaining geographic dewlap variation.

Conclusion: Our study suggests that adaptive dewlap color differences are maintained under strong divergent natural selection, but this divergence does not necessarily lead to anole speciation.
\end{abstract}

Keywords: Dewlap, Geographic variation, Local adaptation, Speciation, Anolis distichus

\section{Background}

Geographic trait variation is widely found within species [1]. The evolution of such variation can arise from divergent natural selection driving adaptation to local environments [2, 3], but whether adaptive trait variation persists through time depends on whether divergent selection is strong enough to counteract the homogenizing effects of gene flow [4-6]. Two alternatives may therefore result from ecologically-driven phenotypic divergence: speciation, whereby reproductive isolation has evolved, or selection may maintain a stable coexistence of two or more locally adapted phenotypes despite gene flow. Identifying the consequence of adaptive divergence is central to understanding the roles of local adaptation and speciation during the early stages of the formation of biological diversity.

\footnotetext{
* Correspondence: julienne.ng@colorado.edu

${ }^{1}$ Department of Ecology and Evolutionary Biology, University of Colorado, Boulder, CO 80309, USA

Full list of author information is available at the end of the article
}

Comparative studies of naturally replicated hybrid zones where adaptively divergent, closely related taxa come into contact and potentially interbreed can provide a powerful approach to study how patterns of introgression and adaptive divergence can vary with geographic space and time [7]. In particular, fine-scaled molecular genetic analyses across such replicated contact zones can directly test whether divergent natural selection necessarily leads to divergence at neutral loci (e.g. [8-11]). For example, studies of mice and lizards that adaptively vary in coloration for crypsis show that not all geographic transitions in color are associated with reduced gene flow $[8,10]$. This suggests that although phenotypically different populations have yet to evolve barriers to reproduction, strong selection is acting to maintain phenotypic divergence, most likely from visual predators [12-14].

A remarkably polymorphic lizard, Anolis distichus, provides an ideal system to investigate whether adaptive trait divergence consistently leads to the same genetic outcome. Across Hispaniola, A. distichus' dewlap (an 
extensible throatfan) varies adaptively in response to heterogeneous environments, whereby dewlaps tend to be larger, orange and less bright in wetter habitats, while in drier habitats, dewlaps are smaller, yellow and relatively brighter [15]. This geographic variation in dewlap color has led taxonomists to diagnose eight subspecies on mainland Hispaniola alone [16] that are characterized by deeply divergent mitochondrial DNA (mtDNA) haplotypes, and differentiation in allozyme and nuclear DNA loci [17-19]. We have also shown in previous work that in two areas of contact, subspecies with different colored dewlaps show a reduction in gene flow, consistent with the expected signature of speciation [20]. However, it remains unclear if these reduced patterns of gene flow occur at other contact zones between divergent dewlap phenotypes, or whether other phenotypic disjunctions represent cases of strong natural selection on dewlap color but with ongoing gene flow. The many other naturally replicated shifts in dewlap color across the range of $A$. distichus provide an excellent opportunity to assess how selection on an adaptive trait may be associated with patterns of introgression across space and time.

Here, we significantly expand upon prior work by conducting replicated molecular genetic analyses of nine transects that span $A$. distichus populations. We contrast results from five geographically diverse transects that extend across phenotypically distinct populations that have been diagnosed using differences in dewlap color and pattern, and four control transects that span the same dewlap color. Specifically, we aim to test whether adaptive dewlap color divergence is associated with (i) speciation, whereby genetic differentiation is observed with neutral markers. Following the general lineage concept $[21,22]$, this result would suggest that populations differing in dewlap color represent independently evolving lineages. Alternatively, (ii) if genetic differentiation is not observed with neutral markers, this suggests that locally adapted traits are maintained in the face of continued gene flow.

\section{Methods}

\section{Sampling}

We sampled nine linear transects in the Dominican Republic: five transects that spanned from populations with primarily yellow dewlaps to populations with primarily orange dewlaps (herein referred to as "transitional" transects; T1a, T1b, T2-T4, Fig. 1), and four control transects that extended across populations exhibiting the same dewlap color (C1-C4, Fig. 1). Four of the five transitional transects spanned populations previously diagnosed as different subspecies, primarily based on dewlap color differentiation [16]. The only exception was transect T4, which spanned populations assigned to a single subspecies (A. d. favillarum) that exhibits geographic variation in dewlap color. All four control transects involved sampling within the range of a single subspecies.

For both transitional and control transects, we aimed to sample five or six evenly spaced localities along 10$20 \mathrm{~km}$ linear transects (Fig. 1, Additional file 1: Table S1). For the transitional transects, this sampling scheme captured the transition in dewlap color from one dewlap color extreme to the other. Inaccessibility or unsuitable habitat determined transect orientation and also led to some variation in distance between sites $(1.74 \mathrm{~km}$ to $8.43 \mathrm{~km}$; mean $\pm \mathrm{SD}=3.26 \pm 1.55 \mathrm{~km}$ ). We previously reported results from sampling along two of these transects, T1a and T2 [20]. Here, however, we include data from an additional site between sites 2 and 3 along transect T2 that appears to be close to the point of contact between the two subspecies (T2-2.5; 0.89 and $1.55 \mathrm{~km}$ from sites 2 and 3 , respectively). In addition, following initial sampling of T3 (T3-4 to T3-8), we subsequently extended the transect into the range of $A$. $d$. ignigularis with three additional sites sampled at larger intervals (T3-1 to T3-3) after preliminary analyses failed to recover evidence for geographic genetic variation. As such, these additional sites ranged from $5.28-19.23 \mathrm{~km}$ in distance from the next adjacent sites, resulting in T3 spanning a total distance of $42.20 \mathrm{~km}$. All control transects spanned populations of the same subspecies and ranged in distance from $8.98-14.03 \mathrm{~km}$, with four or five localities sampled along each transect (Fig. 1, Additional file 1: Table S1). We obtained tissue samples (either tail tips or livers) for molecular genetic analyses from a median of 20 individuals at each transect locality (sample sizes ranged from 10 to 38; Additional file 1: Table S1). In addition to transect sampling, we also sampled 20 individuals from three additional localities (herein called non-transect sites), each of which represent $A . d$. dominicensis, $A$. $d$. ignigularis and $A$. $d$. ravitergum elsewhere within their ranges to further investigate geographic genetic variation (Fig. 1).

\section{Characterizing dewlap color variation}

We ascertained phenotypic variation across all transects by visually categorizing patterns of dewlap color following previous studies $[18,20]$ : (i) primarily yellow $(<10 \%$ orange), (ii) yellow with a small orange spot (occupying $10-40 \%$ of the dewlap), (iii) yellow with a large orange spot (occupying $50-90 \%$ of the dewlap), or (iv) orange with a narrow yellow margin ( $>90 \%$ orange). We further categorized any orange coloration as either diffuse (blush) or solid. Assignments of each dewlap were independently assessed and verified by JN and REG. Although we focus on visual assessments of dewlap color (as yellow or orange), this has previously been shown to be consistent with spectrometer measurements of hue, which is an axis of adaptive dewlap divergence $[15,20]$. In addition, while some $A$. distichus dewlaps 


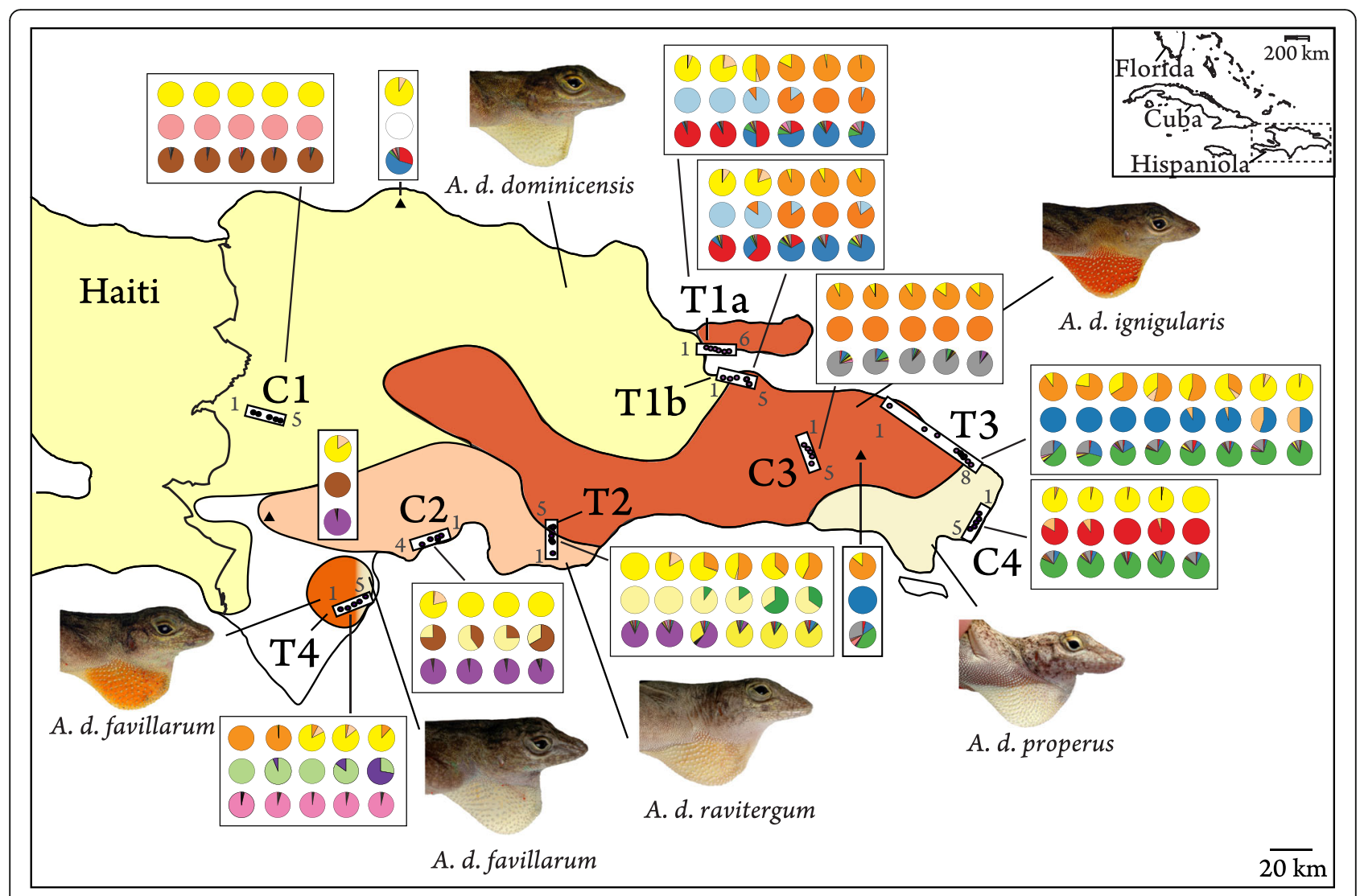

Fig. 1 Distribution and sampled localities of Anolis distichus subspecies in the Dominican Republic. Colored regions demark respective range distributions and dominant dewlap color of $A$. distichus subspecies (adapted from [16]). Filled black circles surrounded by boxes represent the study transects and associated sampling localities: T1 - T4 represent transects that transition between populations with different dewlap coloration; C1 - C4 represent control transects that span populations with similar dewlap coloration. To permit comparison to other figures, the first and last sample sites of each transect are numbered. Black triangles indicate additional sampled sites outside of our transects. Pie charts represent dewlap and genetic variation for each site in numerical order from left to right (see also Fig. 2 for more details). The top row of pie charts represent dewlap color variation with yellow, orange and peach proportions indicating the average proportion of yellow, and solid or diffuse orange visually observed in the dewlap, respectively. Females or juveniles found at the site are not included as they cannot be scored for dewlap color. The middle row of pie charts represents mitochondrial haplotype variation as determined from a maximum likelihood phylogenetic analysis, with different colors representing different mitochondrial clades (see also Fig. 3). Missing haplotype data is not included. The bottom row of pie charts represents nuclear genotypic variation as determined from STRUCTURE analyses, with different colors representing different genetic clusters ( $K$ ) ( $K=9$ was the best estimate) (see also Fig. 2 for more details). Further substructure from subsequent STRUCTURE analyses on separate clusters is not shown

reflect ultraviolet (UV) wavelengths, UV reflectance is not correlated with different habitat types and is therefore not considered an adaptive trait in this species [15]. Juveniles and females, which lack or have reduced dewlaps, were not scored for dewlap color.

\section{Characterizing genetic variation}

\section{Mitochondrial DNA sequencing and phylogenetic analyses}

We investigated patterns of mtDNA differentiation along transects by obtaining mtDNA sequence data for all individuals sampled from sites along each transect $(N=$ 955). We focused on a 1147 basepair (bp) region of mtDNA extending from the beginning of ND2 (subunit two of NADH dehydrogenase) through to tRNA ${ }^{\text {Ala }}$ that has been widely used in previous phylogenetic and phylogeographic studies of anoles (e.g. [23-29]). We also obtained mtDNA sequence data from this same region for individuals at the three non-transect sites as well as 195 additional individuals from 50 other $A$. distichus populations across Hispaniola, including three Hispaniolan subspecies that did not occur in any of our transects: A. d. sejunctus, a subspecies found on Isla Saona (an island off the south-east coast of the Dominican Republic), and two Haitian subspecies, $A . d$. vinosus and $A$. $d$. aurifer. To represent outgroups, we obtained previously published mtDNA haplotypes from eight species: four species from the $A$. distichus species group (A. brevirostris, $A$. caudalis, A. websteri and A. marron), two representatives from clades closely related to the distichus series (A. cristatellus [cristatellus series] and A. bimaculatus 
[bimaculatus series]) [30] and two more distantly related anoles (A. punctatus and A. occultus) [27]. Our complete mtDNA dataset comprised haplotypes sampled from 1305 individuals, of which 675 were unique; 332 sequences were obtained from previous studies [15, 17, 20, 27] and 973 new sequences were generated for this study (GenBank accession numbers: KX854021-KX855205).

We generated new mtDNA sequences by first extracting genomic DNA from tissue samples using the Wizard genomic DNA purification kit (Promega). Using primers L4437 [31] and H5730 [32], we conducted $25 \mu \mathrm{L}$ PCR reactions with $1 \mu \mathrm{L}$ genomic DNA, $2.5 \mu \mathrm{L}$ of each $2 \mu \mathrm{M}$ primer, $2.5 \mu \mathrm{L}$ of New England Biolabs (NEB) 10× buffer $(10 \mathrm{mM}$ Tris- $\mathrm{HCl}, 50 \mathrm{mM} \mathrm{KCl}), 2.5 \mu \mathrm{L}$ of $25 \mathrm{mM}$ $\mathrm{MgCl}_{2}, 2.5 \mu \mathrm{L}$ of $0.5 \mathrm{mM}$ dNTP solution, $0.125 \mu \mathrm{L}$ of NEB Taq polymerase and $11.4 \mu \mathrm{L}_{2} \mathrm{O}$. Our thermocycling protocol involved an initial denaturation at $95{ }^{\circ} \mathrm{C}$ for $4 \mathrm{~min}$, followed by 30 cycles of $95{ }^{\circ} \mathrm{C}$ for $35 \mathrm{~s}$, annealing at $52{ }^{\circ} \mathrm{C}$ for $35 \mathrm{~s}$, and extension at $70{ }^{\circ} \mathrm{C}$ for 2 min $30 \mathrm{~s}$. We sent successfully amplified PCR products to a commercial facility for purification and Sanger sequencing (Beckman Coulter Genomics, Massachusetts). We assessed each sequence chromatograph for quality in Geneious v4.6.1 [33] and used the pairwise alignment tool in MacClade 4.0 [34] to align sequences. With no indels in the protein coding sequences, alignment was straightforward. The tRNA genes were aligned using secondary structural models [35]. As $43 \mathrm{bp}$ of sequence data from tRNA genes were highly variable in length and a region of apparent ambiguous alignment, we excluded these regions from our dataset prior to analysis, leaving 1104 aligned characters without gaps.

We inferred phylogenetic relationships among $A$. distichus haplotypes using a maximum likelihood (ML) approach in RAxML v7.0.3 [36] and Bayesian inference in MrBayes v3.2 [37, 38] (alignment and trees deposited in TreeBASE: study ID S19838). We used PartitionFinder v1.1.1 [39] to determine the best fitting partitioning scheme with four data blocks: a separate partition for each codon position within ND2 and a fourth partition for the tRNA region. For RAxML, PartitionFinder identified three partitions to be the best fitting scheme, with GTR + I + G to be the best fitting substitution model for the first ND2 codon position and the tRNA region merged as one partition, as well as the second ND2 codon position, while GTR $+\mathrm{G}$ was identified as the most appropriate model of evolution for the third ND2 codon position region. For MrBayes, which allows analyses under more evolutionary models, PartitionFinder identified $\mathrm{HKY}+\mathrm{I}+\mathrm{G}$ to be the most appropriate model of evolution for the first ND2 codon position, GTR + I + $G$ for the second ND2 codon position and tRNA region, and GTR $+\mathrm{G}$ for the third ND2 codon position. For both ML and Bayesian analyses, we did not include a parameter for the proportion of invariable sites (I) since the interactions between the I and G parameters can potentially lead to inaccurate estimates due to nonindependent optimization [40]. For our ML analysis, we conducted 1000 nonparametric bootstrap replicates and computed pairwise ML patristic distances from the best ML tree. For trees generated under Bayesian inference, we ran two independent analyses for 10 million generations and assessed convergence by checking the average standard deviation of split frequencies in MrBayes and using Tracer v1.6 [41]. We considered there to be genetic differentiation in mitochondrial DNA along our sampled transects if populations from either ends of the transect belonged to distinct mitochondrial clades (node support as measured by bootstrap values and posterior probabilities $\geq 75 \%$ ).

For each transect site sampled, we calculated the number of haplotypes, number of polymorphic sites, nucleotide diversity and the mean number of pairwise differences between haplotypes using ARLEQUIN v3.11. Using the same program, we also assessed genetic differentiation between populations using $\Phi_{\mathrm{ST}}$ (an analogue of $\mathrm{F}_{\mathrm{ST}}$ ) and tested for significant genetic differences between sites using 10,000 permutations. We also constructed a haplotype network that comprised haplotypes from all transect sites in PopART (http://popart.otago. ac.nz) using the TCS method [42].

\section{Microsatellite genotyping and population structure analyses}

To assess nuclear differentiation along transects, we genotyped all individuals using 7 highly polymorphic di- and trinucleotide microsatellite loci: DISTA2B12, DISTBB5, DISTBC4, DISTAG1, DISTCC7, DISTAH6 and BREV2E9 [43]. We followed the same PCR protocols as described for mtDNA sequence data (above section) but, following $\mathrm{Ng}$ and Glor [20], we included a fluorescently-labelled CAG primer (6-FAM, VIC or NED) at a 1:20 ratio $(0.01 \mu \mathrm{M}$ CAG-tagged forward primer and $0.19 \mu \mathrm{M}$ fluorescent CAG primer) to enable multiplexing. We followed thermocycling conditions detailed in $\mathrm{Ng}$ et al. [43] and visualized products on an ABI 3730 Genetic Analyzer (Applied Biosystems) located at the University of Rochester's Functional Genomics Center. Genotypic data were analyzed with GENEMAPPER V3.7 software (Applied Biosystems). We tested all microsatellite loci for within population departures from Hardy-Weinberg equilibrium (HWE) and linkage disequilibrium (LD) using GENEPOP v4.0 [44], and accounted for multiple tests using sequential Bonferroni correction [45].

We tested for nuclear genetic differentiation along each transect using (i) genetic distance metrics and (ii) a Bayesian clustering approach. We did not use cline analyses (e.g. $[46,47]$ ) because our microsatellite markers 
are not diagnostic; they do not show fixed, or almost fixed, differences between the two subspecies along transects (see Results). We calculated pairwise estimates of $\Phi_{\text {ST }}$ between adjacent sites along each transect using ARLEQUIN v3.11 and tested for significant genetic differences between sites using 10,000 permutations. In addition, we used a genetic distance metric based on the proportion of shared alleles between populations $\left(D_{\mathrm{PS}}\right)$ [48], calculated using MSA v4.05 [49]. $\mathrm{D}_{\mathrm{PS}}$ has an advantage over $\Phi_{\mathrm{ST}}$ in that it does not assume population equilibrium [50, 51].

We conducted Bayesian clustering analyses using the program STRUCTURE v2.3.3, which uses a Markov chain Monte Carlo algorithm to probabilistically assign individuals to different genetic clusters $(K)$ [52]. We inferred genetic structure after combining data across all transects and the three non-transect sites. We used the admixture model, which allows individuals to have mixed ancestry, in addition to the correlated allele frequencies model, which accounts for potential linkage disequilibrium between markers that can arise due to admixture [53], and did not include a priori information about where individuals were sampled. We ran analyses for $10^{5}$ iterations following a burn-in period of $10^{4}$ iterations for each value of $K$ ranging from $1-15$. We chose a maximum $\mathrm{K}$ value of 15 because we expected that there would be at least 8 different genetic clusters across our transitional transects if genetic differentiation is associated with adaptive dewlap color transition (or at least 10 if individuals of the same subspecies were genetically different along $\mathrm{T} 1 \mathrm{a}$ and $\mathrm{T} 1 \mathrm{~b})$. We ran 10 independent runs of $K$ to ensure consistent probability estimates and that particular runs were not trapped in different modes in the parameter space [52]. The average of all ten runs were subsequently used to assess genetic structure. To identify the best estimate of $K$, we used log probabilities $\operatorname{Pr}(\mathrm{X} \mid K)$ and calculated $\Delta K[54]$ using the web-based program STRUCTURE HARVESTER [55]. We also investigated whether there was more subtle population structure by repeating the analysis on each genetic cluster identified. Following protocols outlined in Coulon et al. [56], we assigned individuals to a particular genetic cluster if their inferred ancestry to that group was higher than 0.6. With each genetic cluster, we sequentially ran STRUCTURE analyses using the same parameters as the initial run and continued this process until the optimal $K$ was 1.

We interpreted results of distinct nuclear genotypic clusters at either ends of the transect to be evidence for neutral genetic divergence. We diagnosed how far along populations were in the speciation continuum by the frequency of admixed individuals. If geographic genetic structure was evident but the frequency of admixed individuals was high and widely spread across different populations, we considered this to reflect low levels of restricted gene flow, and that the populations were at an earlier stage in the speciation continuum. In contrast, a limited number or no evidence of admixed individuals indicated a strong reduction of gene flow, likely representing populations in the later stages of speciation.

\section{Results \\ Dewlap color variation}

Along all transitional transects, we confirmed the presence of a phenotypic transition in dewlap color and pattern. At one side of each transect, males exhibited dewlaps that were completely yellow or had a small proportion of orange, while towards the other side of the transect, we found males with a higher proportion of orange in their dewlaps (Figs. 1 and 2). The nature of the shift between yellow and orange dewlapped populations, however, varied among transitional transects. For example, T1b and T4 exhibited an abrupt shift in the proportion of orange found in the dewlap; populations characterized by largely yellow or largely orange dewlaps were separated by only approximately $3 \mathrm{~km}$ and there was little evidence for intermediate amounts of orange at any of the sampled localities (Figs. 1 and 2). Meanwhile, T1a and T3 exhibited a more gradual cline in the amount of orange in the dewlaps; populations having almost entirely orange or yellow dewlaps were separated by 6.65 and $20.58 \mathrm{~km}$ respectively, and one or more localities included individuals with intermediate amounts of orange in their dewlaps (Figs. 1 and 2). Along the remaining transitional transect, T2, dewlaps were rarely entirely or almost entirely orange, but the presence of some orange in the dewlap transitioned from being uncommon to common over a relatively short distance $(0.89 \mathrm{~km})$ (Figs. 1 and 2$)$.

We found similar dewlap phenotypes across each control transect. All males sampled along C3 had dewlaps with greater than $60 \%$ orange, while individuals sampled along all other control transects had very little orange in the dewlaps; the two individuals at $\mathrm{C} 2-1$ that exhibited greater than $50 \%$ orange in the dewlap was diffuse in coloration (Figs. 1 and 2).

\section{Mitochondrial phylogenetic relationships}

Phylogenetic reconstructions under ML and Bayesian inference were largely congruent with node support greater or equal to $75 \%$ for major groups (Fig. 3), with the exception that the ML tree showed strong support for a clade comprising $A$. d. ignigularis haplotypes from $\mathrm{T} 1 \mathrm{a}, \mathrm{T} 1 \mathrm{~b}$ and $\mathrm{C} 3$, while the Bayesian consensus tree showed strong support for a separate clade for each transect. As this incongruence does not affect our overall conclusions, we here only discuss and report clade membership for $A$. $d$. ignigularis based on to the ML tree for brevity. 


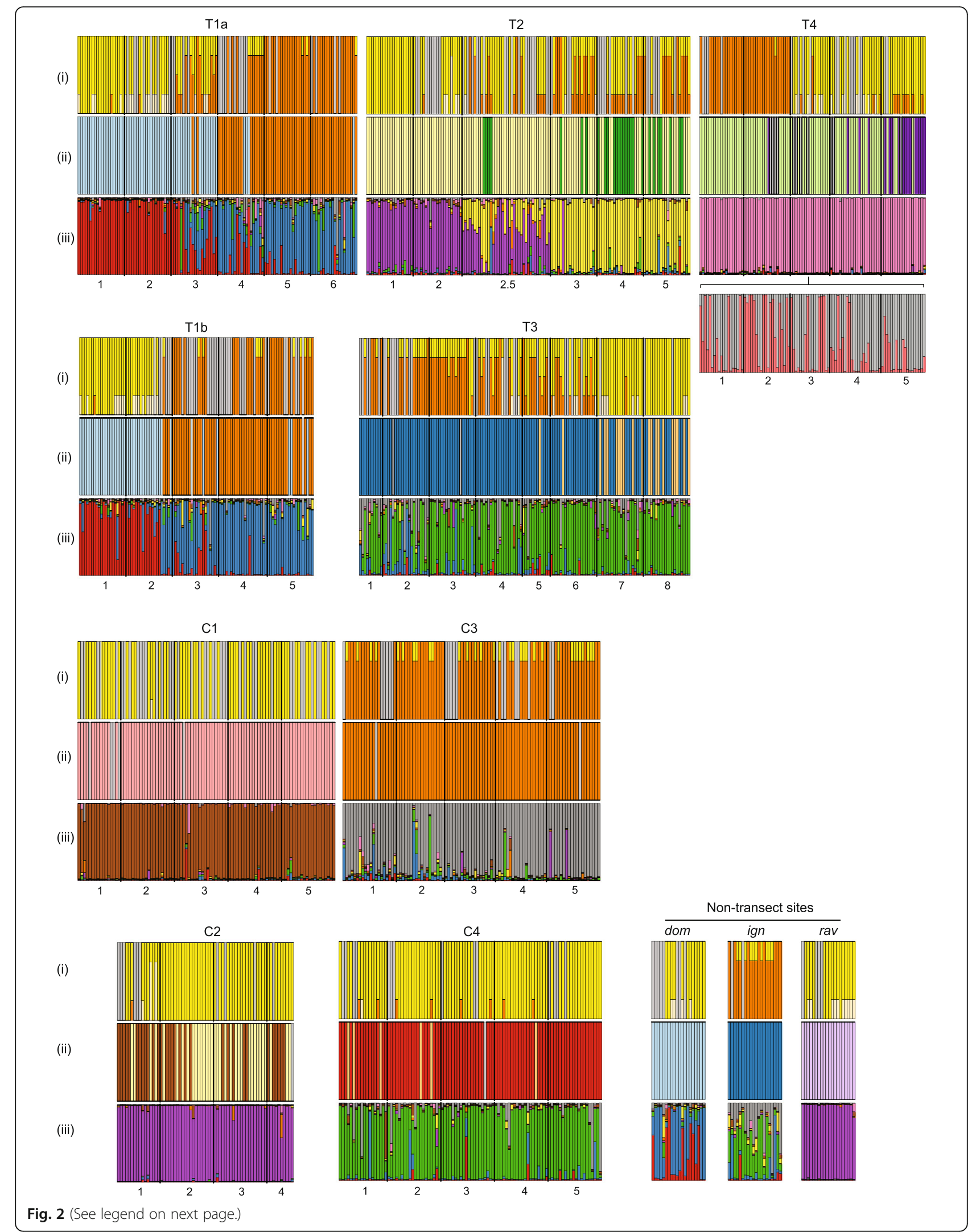


(See figure on previous page.)

Fig. 2 Phenotypic and genetic variation across transitional transects (T1-4), control transects (C1-4) and non-transect sites. Vertical bars in each graph represent individuals and vertical black lines demark different sample sites. Sample site numbers are shown underneath each set of graphs. i Dewlap color variation with colors representing percentages of orange and yellow in the dewlap. Peach colored bars represent a diffuse orange while grey bars represent females or juveniles, which lack or have reduced dewlaps and thus were not scored for color. ii Mitochondrial haplotype variation as determined from a maximum likelihood phylogenetic analysis. Different colored bars represent haplotypes belonging to different clades (see also Fig. 3). Grey bars represent missing haplotype information. iii Nuclear genotypic variation as determined from STRUCTURE analyses $(K=9)$. Each color represents a distinct genetic cluster, with different colors within each bar representing the proportion of the individual's genotype assigned to the cluster. Proportions shown are averages from 10 replicate runs. The additional graph shown under T4 indicates substructure revealed with additional STRUCTURE analyses following [56]

Along each transitional transect, we found haplotypes from two different mitochondrial clades (Figs. 1, 2 and 3). Along T1a and T1b, most of the haplotypes sampled from either end of the transects showed deep divergence; haplotypes along both $\mathrm{T} 1$ transects belonged to deeply divergent clades $(15.1 \%$ and $15.8 \%$ average uncorrected sequence divergence, respectively) and were also divergent in the haplotype network (Additional file 1: Figure S1). Haplotypes from the western side of the transects grouped with other $A$. $d$. dominicensis haplotypes while haplotypes from the eastern side grouped with other A. d. ignigularis haplotypes (Fig. 3). This differentiation was also reflected in significant $\Phi_{\mathrm{ST}}$ estimates, particularly between sites T1a-3 and $4\left(\Phi_{\mathrm{ST}}=\right.$ $0.75 ; P<<0.001)$ and sites T1b-2 and $3\left(\Phi_{\mathrm{ST}}=0.60 ; P<\right.$ $<0.001)$ which were each at least 4 times greater than any other adjacent sites along the same transect, after correcting for differences in geographic distance (Fig. 4). A few haplotypes sampled from the middle and more eastern sites were assigned to the same clade of $A . d$. dominicensis haplotypes as the haplotypes sampled from the westernmost sites, suggesting limited mtDNA introgression (Figs. 1 and 2). The middle sites of T1a and b also had greater mtDNA genetic diversity (Additional file 1: Table S2). All other transitional transects (T2-T4) exhibited relatively less genetic differentiation in mtDNA (T2-T4: $3.7 \%, 4.0 \%$ and $5.0 \%$ average uncorrected sequence divergence, respectively) as well as evidence of mtDNA introgression (Figs. 1 and 2). Mitochondrial genetic diversity estimates reflected these patterns whereby we found greater mtDNA haplotype and nucleotide diversity at sites that showed mtDNA introgression (Additional file 1: Table S2).

Along the control transects, $\mathrm{C} 1$ and $\mathrm{C} 3$ comprised mtDNA haplotypes from the same clade (Figs. 1, 2 and 3). We found haplotypes from two closely related clades along C2 and C4 (4.7\% and $5.3 \%$ average uncorrected sequence divergence, respectively) but these haplotypes did not show any geographic structure (Figs. 1 and 2).

Assessing overall phylogenetic relationships among $A$. distichus mtDNA haplotypes, we found $A$. d. favillarum to be the only monophyletic Dominican subspecies from mainland Hispaniola. Mitochondrial haplotypes from the other four Dominican $A$. distichus subspecies from mainland Hispaniola (A. d. dominicensis, A. d. ignigularis, $A . d$. properus and $A . d$. ravitergum) did not form monophyletic groups (Fig. 3 ). Instead, $A$. d. ravitergum and $A . d$. properus sampled along our transects were each rendered paraphyletic by $A$. $d$. ignigularis sampled along the same transects (T2 and T3 respectively). Our finding that most $A$. distichus subspecies are nonmonophyletic is inconsistent with other phylogenetic studies of the group [17, 19], but is likely due to our inclusion of individuals from subspecific contact zones where mitochondrial introgression can confound phylogenetic placement. Our phylogeny, however, is consistent with Geneva et al. [19] in showing well-supported haplotype clades within $A . d$. dominicensis that appear to be geographically structured; haplotypes from transect $\mathrm{C} 1$ in central Hispaniola are most closely related to haplotypes sampled from $A$. d. favillarum and Haitian A. distichus subspecies, while $A$. $d$. dominicensis haplotypes sampled from northern Dominican Republic (T1a and b, non-transect $A$. d. dominicensis site) formed a separate group (Fig. 3).

\section{Microsatellite loci tests of HWE and LD}

The microsatellite loci exhibited significant deviations from HWE in 57 of 364 intrapopulation assessments (Additional file 1: Table S1). All loci but DISTAH6 deviated from HWE in only a few populations (3-10 populations), while DISTAH6 deviated from HWE in 23 of 57 sample sites. Thus, we ran analyses using all seven loci and then repeated analyses without genotypic data from DISTAH6. As analyses did not qualitatively differ, we here report results of analyses on all seven loci. We detected little evidence of LD among microsatellite loci; in 6 of 52 sampled sites, one pair of loci showed significant LD after Bonferroni correction $(P<0.007)$. These pairs differed between sites, except for DISTCC7 and DISTAH6, which showed significant LD in two sites along T4 (T4-1 and T4-3).

\section{Patterns of nuclear genetic structure}

The results of our STRUCTURE analysis identified $K=9$ to be the best estimate of $K$. Of the transitional transects, three showed that individuals on either side of the transect belonged to different genetic clusters (T1a and 


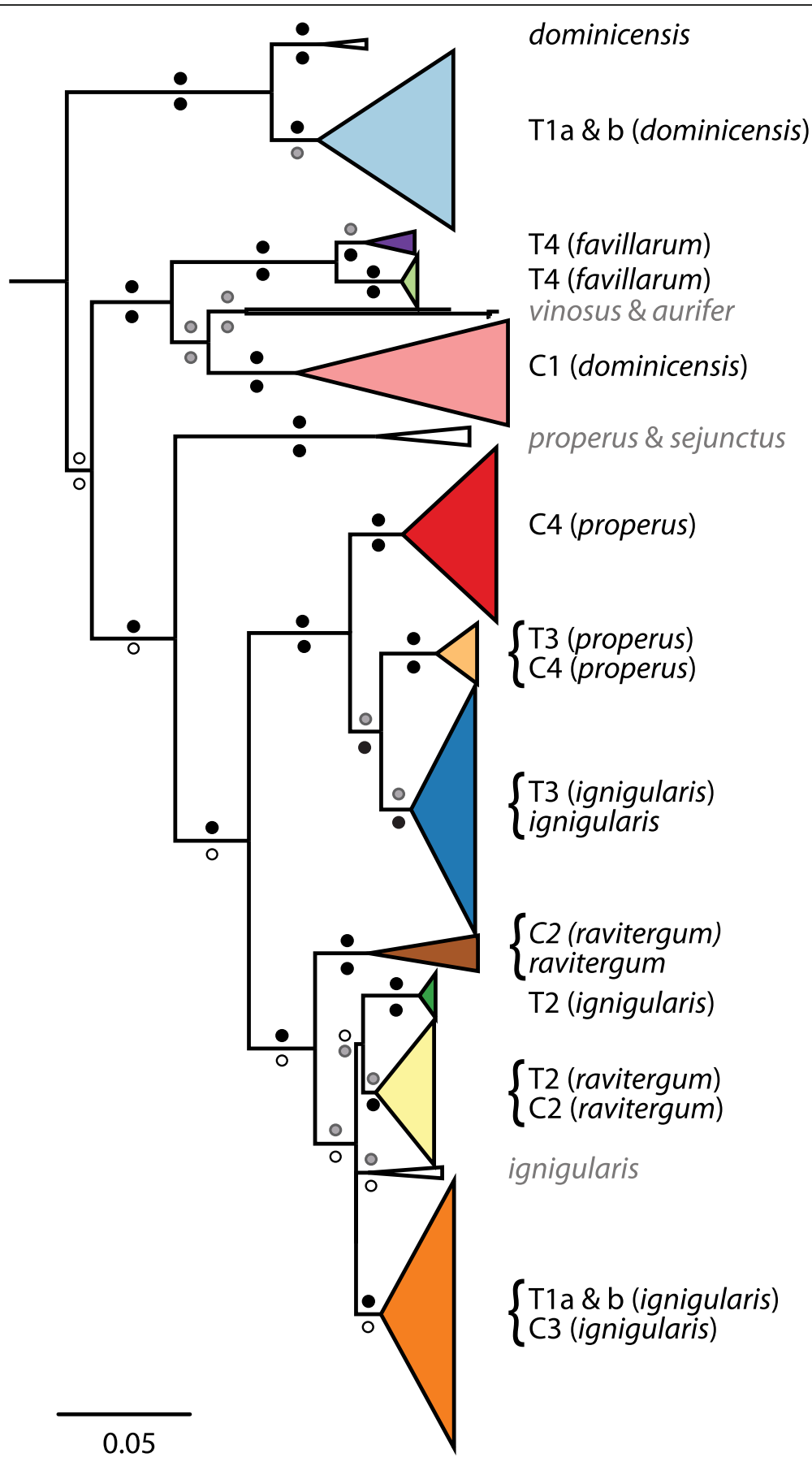

Fig. 3 Maximum likelihood phylogeny inferred by RAxML from Anolis distichus mtDNA haplotypes. Haplotypes used as outgroups have been pruned from this tree. Colored triangles represent clades and are labelled with the transect and subspecies from which the haplotype was sampled (note that for transitional transects, the expected subspecies collected is listed based on locality along the transect). Labels in black font without transect IDs represent non-transect sampled sites. Clades labelled in grey font represent haplotypes that were sampled from additional sites that were included in the phylogenetic analysis but not used in other aspects of this study. Circles above and below branches represent node support measured as bootstrap values and posterior probabilities from RAxML and MrBayes, respectively: black $\geq 90 \%$, grey $\geq 75 \%$, white $<75 \%$

b, T2; Figs. 1 and 2). Sampled sites in the middle of these transects included individuals from both genetic clusters as well as admixed individuals. While at least three localities included admixed individuals along T1a and $\mathrm{T} 1 \mathrm{~b}$, we only found admixed individuals at one site along T2 (T2-2.5). The other two transitional transects 


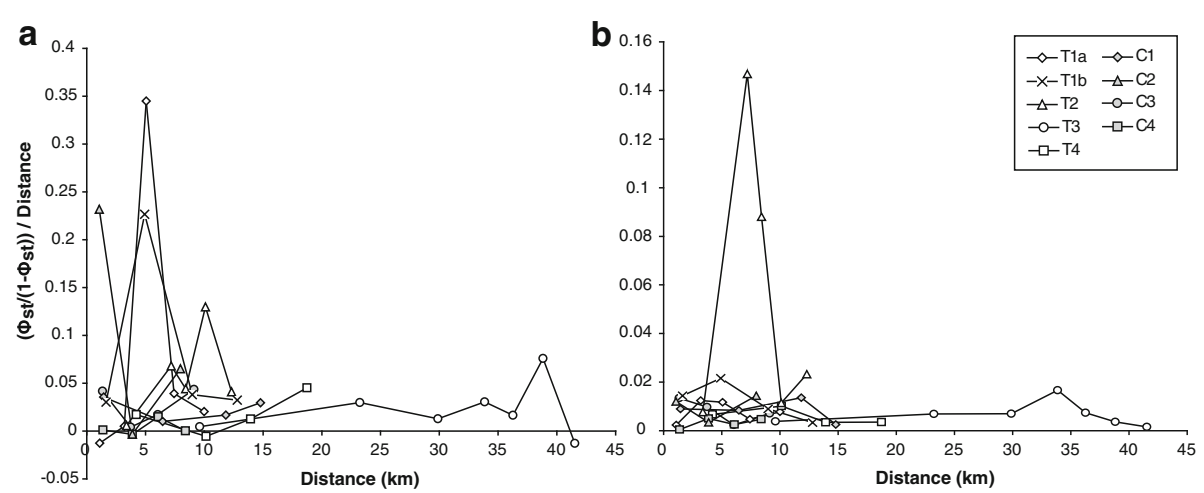

Fig. 4 Pairwise estimates of $\Phi_{S T}$ in (a) mtDNA and (b) microsatellite loci between sites sampled along transitional transects (T1-T4; unfilled shapes) and control transects (C1-C4; filled shapes). $\Phi_{\text {ST }}$ estimates were adjusted for distance between sites using linearized genetic distance $\left(\Phi_{S T} /\left(1-\Phi_{S T}\right)\right)$ divided by geographic distance $(\mathrm{km})$

(T3 and T4) did not show any genetic structure despite T3 being the longest transect; most individuals sampled along the transect were predominantly assigned to the same cluster. Additional runs on each genetic cluster revealed two further genetic clusters along T4 but there was no obvious geographic structuring (Fig. 2). Individuals along control transects were primarily assigned to the same genetic cluster as others sampled from the same transect (Fig. 2).

While STRUCTURE analyses showed T1a, T1b and T2 to all exhibit genetic differentiation, pairwise $\Phi_{\mathrm{ST}}$ and $\mathrm{D}_{\mathrm{PS}}$ estimates showed that genetic differentiation along $\mathrm{T} 1 \mathrm{a}$ and T1b were comparable to that found along the control transects and the transitional transects, T3 and T4, which did not exhibit genetic structure in the STRUCTURE analyses (Fig. 4, Additional file 1: Figure S2). Meanwhile, pairwise $\Phi_{\mathrm{ST}}$ and $\mathrm{D}_{\mathrm{PS}}$ estimates along T2 showed moderately high genetic differentiation between $\mathrm{T} 2-2$ and $\mathrm{T} 2-2.5\left(\Phi_{\mathrm{ST}}=0.12 ; \mathrm{D}_{\mathrm{PS}}=0.48\right)$ that, after correcting for differences in geographic distance, were at least 7 and 3 times greater respectively than that found along any other transect (geographic distance corrected: $\Phi_{\mathrm{ST}}=0.15 ; \mathrm{D}_{\mathrm{PS}}=0.54 ;$ Fig. 4 , Additional file 1: Figure S2).

Assessing general genetic structure within subspecies, we found that subspecies that have a widely distributed range exhibited nuclear genetic structure. The most widespread of the subspecies, $A$. d. dominicensis, comprised two clusters: one cluster comprised the control transect $(\mathrm{C} 1)$ while the other included some T1a and b populations (Figs. 1 and 2). The non-transect $A$. $d$. dominicensis site comprised individuals, or proportions of individuals, assigned to either one of the two clusters found along T1a and $\mathrm{b}$. We found three clusters within A. d. ignigularis: one cluster included some sites along $\mathrm{T} 2$, one included some sites sampled along $\mathrm{T} 1 \mathrm{a}$ and $\mathrm{b}$, while the other included the $A . d$. ignigularis control transect (C3) (Figs. 1 and 2). The non-transect $A$. $d$. ignigularis site looked similar to sites found along T3, whereby it comprised individuals, or proportions of individuals, assigned to either cluster found along C3 and $\mathrm{C} 4$. All $A$. $d$. ravitergum sampled were initially assigned to the same genetic cluster (T2, C2 and the non-transect $A$. d. ravitergum site) (Figs. 1 and 2). Additional STRUCTURE analyses showed further substructure into two clusters: C2 comprised one cluster, while $\mathrm{T} 2$ and the non-transect site were assigned to another cluster (results not shown). Further STRUCTURE analyses differentiated T2 $A$. $d$. ravitergum from the non-transect site.

\section{Discussion}

Our study examining numerous transitions in adaptive dewlap coloration in Anolis distichus across the Dominican Republic suggests that adaptive trait divergence is associated with both speciation and local adaptation in the face of gene flow. We find evidence for local adaptation at two transects (T3 and T4), whereby there was no evidence of genetic differentiation despite bimodal phenotypic variation. Patterns of mtDNA variation suggest that introgression is occurring and nuclear variation at microsatellite loci does not show evidence of any genetic structure. This is further supported by pairwise estimates of $\Phi_{\mathrm{ST}}$ and $D_{\mathrm{PS}}$ that indicate similar levels of pairwise genetic differentiation to that found along the control transects. Despite the homogenizing effects of gene flow, the maintenance of dewlap color variation at such a fine spatial scale suggests that strong divergent natural selection is acting on loci underlying dewlap color differences between spatially close sites while neutral regions of the genome are homogenized with gene flow. This also suggests that the genes underlying dewlap color likely have different evolutionary trajectories that is not reflected by the results of our analyses of 
neutral markers. Together, these results support the importance of divergent natural selection, rather than neutral processes, in driving and maintaining dewlap color variation along these transects. These transects are similar to previous studies that have shown that adaptive trait divergence between populations can be maintained in the face of gene flow (e.g. [57-60]), including cases of adaptive color divergence $[8-10,61,62]$. It is also possible that while we did not find evidence of genetic differentiation with our markers, such ecologically-based trait divergence may represent the initial step towards speciation [63-66].

We find patterns consistent with the expected signature of speciation along three transects (T1a, T1b and T2) whereby adaptive dewlap color divergence is associated with genetic differentiation. The results of our control transects, showing no genetic differentiation where there was no change in dewlap color, further support the possibility of speciation occurring at these three transitional transects. The transects, however, exhibited differing levels of genetic differentiation and therefore likely represent different stages along the speciation continuum. T1a and b appear to be at an earlier stage along the speciation continuum relative to T2. Along T1a and b, phylogenetic and STRUCTURE analyses show distinct mtDNA clades and nuclear genetic clusters on either side of the transect, respectively. Both mtDNA and nuclear markers, however, suggest a low reduction in gene flow between the phenotypically divergent populations: mtDNA haplotypes prevalent on the western side of the transect were still found at a low frequency in the middle and other side of the transect, and nuclear markers showed evidence of admixed genotypes that decreased in frequency from the middle of the transect to the east. Furthermore, pairwise genetic distance estimates $\left(\Phi_{\mathrm{ST}}\right.$ and $\mathrm{D}_{\mathrm{PS}}$ ) exhibit similar levels to that found along transects that show no genetic differentiation (T3, T4 and all control transects). Mitochondrial haplotype structure has been shown to reflect historic geological events while the more quickly evolving microsatellite loci are more likely to reflect contemporary gene flow [11, 67-69]. As such, the deeply divergent haplotypes we sampled from either side of the transects suggest hybridization upon recent secondary contact between $A$. d. dominicensis and $A$. d. ignigularis. While we know that these two subspecies along T1a were once isolated by a seawater channel that separated the Samaná Peninsula from mainland Hispaniola $[20,70]$, we are unaware of any historical barrier that isolated subspecies along $\mathrm{T} 1 \mathrm{~b}$.

Closer to the other end of the speciation continuum, T2 exhibits evidence of a stronger reduction in nuclear gene flow; Bayesian clustering analyses suggest that hybridization is limited to one site (T2-2.5) whereby both parental genotypes and admixed individuals co- occur, and pairwise distance estimates between T2-2 and T2-2.5 are at least 3 times higher than that found along any other transect, despite closely related mtDNA haplotypes showing evidence of introgression. This pattern was also shown in $\mathrm{Ng}$ and Glor [20], but evidence of hybridization was only found with the additional sampling we include in this study. Our results showing high levels of genetic differentiation at nuclear loci along T2 is further supported by a previous phylogenetic reconstruction based on seven nuclear genes that showed that the same two subspecies collected at some of the same localities each formed monophyletic groups [19]. Together, this suggests that the two subspecies along T2 represent independently evolving lineages. The maintenance of a narrow hybrid zone, with no evidence of hybrids at T2-2 or T2-3 $(0.89$ and $1.55 \mathrm{~km}$ away, respectively) suggests strong intrinsic or extrinsic selection against hybrids and almost complete reproductive isolation between $A . d$. ravitergum and $A$. d. ignigularis.

While three transects support the predictions of speciation, it remains unknown whether any of the transitional transects we investigated in this study will progress to speciation, or whether the current stages we observe represent stable states of migration-selection equilibria [71]. The presence of admixed individuals at all transitional transects suggest that some level of gene flow is still occurring, and whether they progress to speciation will likely depend on the strength of divergent selection on dewlap coloration and/or whether divergent selection is also acting on other traits involved with fitness ('multifarious selection') (reviewed in [72]). Given that the degree to which dewlap color diverged across transects did not correlate with genetic divergence, it is likely that other traits are also involved in reproductive isolation and speciation in $A$. disitchus and that the dewlap does not serve as a 'magic trait' [66, 73]. For example, the abrupt reduction in gene flow across T2 was accompanied by a gradual shift in dewlap color, while T4, which showed the most abrupt shift in orange in the dewlap, did not show any genetic structure. Instead, our evidence of some genetic differentiation along T1a, b and $\mathrm{T} 2$, suggests that the reduction in gene flow may be more directly associated with another adaptive trait not quantified in this study. One such trait could be scale differences, which were also used to designate $A$. distichus subspecies [16]. Differences in scale number or size has been shown to vary within and among other anole species, and may represent adaptive divergences to regulate water loss or body temperature in different habitats [62, 74, 75]. If weak selection on multiple traits can promote speciation [76], this may be one reason why the polymorphic A. d. favillarum along T4, which only differs in dewlap color, does not show genetic structure, despite exhibiting the most abrupt dewlap color shift of all transects. 


\section{Conclusions}

Our analysis of multiple replicates of dewlap color divergence in $A$. distichus showed that geographic variation in adaptive dewlap color is associated with both local adaptation and speciation. At some transitional transects, there appears to be strong selection maintaining adaptive dewlap coloration in the face of gene flow. At other contact zones, we found signatures of speciation whereby adaptive dewlap divergence is associated with genetic differentiation, indicating some reduction in gene flow across the transects. Our results are consistent with previous work suggesting that within-island speciation in anoles is limited to the four large Greater Antillean islands [77]. Despite striking adaptive variation in head, body and dewlap coloration in anoles on the smaller islands of the Lesser Antilles, there are no examples of phenotypically divergent populations on these islands that show as strong a reduction in gene flow as we have found between $A$. d. ravitergum and $A$. d. ignigularis across T2 $[9,11,68]$.

\section{Additional file}

\section{Additional file 1: Figure S1. Mitochondrial haplotype network} comprising haplotypes from all transect sites. Figure S2. Pairwise estimates of $D_{P S}$ in microsatellite loci between sites sampled along transitional transects (T1-T4; unfilled shapes) and control transects (C1-C4; filled shapes). DPS estimates were divided by geographic distance $(\mathrm{km})$ to correct for differing distances between sites. Table S1. Descriptions of the transitional transects, control transects and non-transect sites sampled for this study. For each transect, the Anolis distichus subspecies sampled, the length (calculated as distance from first to last site) and the number of study sites are reported. Abbreviations for $A$. distichus subspecies are as follows: $d o m=$ dominicensis, ign = ignigularis, prop = properus, rav = ravitergum, fav = favillarum. For each study site, the number of sampled individuals and observed $\left(H_{0}\right)$ and expected $\left(H_{E}\right)$ heterozygosities are described. Bold text indicates significant departures from Hardy-Weinberg equilibrium after Bonferroni correction $(P<0.007)$. Table S2. Estimates of mitochondrial genetic diversity for each sampled transect population. Subspecies abbreviations are as described in Table S1. (DOC 2030 kb)

\section{Acknowledgements}

We are grateful to Daniel Scantlebury, Anthony Geneva and Miguel Landestoy for their help collecting field samples, and Jorge Brocca for help obtaining permits. We thank members of the Glor Lab and Robert Laport for helpful comments on an early draft of this manuscript.

\section{Funding}

This work was supported by a National Science Foundation grant to R.E.G. (NSF-DEB 0920892).

\section{Availability of data}

All DNA sequence data that was newly generated for this study has been deposited into GenBank (accession numbers KX854021-KX855205). The DNA sequence alignment file and phylogenetic trees reported in this study have been deposited into TreeBASE (study ID S19838).

\section{Authors' contributions}

JN and REG designed and coordinated the study. JN, AGO and REG contributed to data collection and analyses. JN and REG drafted the manuscript. All authors read and approved the final manuscript.

\section{Competing interests}

The authors declare that they have no competing interests.

\section{Animal ethics}

Tissue samples were collected with the authorization of the Ministerio de Medio Ambiente y Recursos Naturales in the Dominican Republic.

\section{Author details}

${ }^{1}$ Department of Ecology and Evolutionary Biology, University of Colorado, Boulder, CO 80309, USA. ²Department of Biology, University of North Georgia, Gainesville, GA 30503, USA. ${ }^{3}$ Biodiversity Institute and Department of Ecology and Evolutionary Biology, University of Kansas, Lawrence, KS 66045, USA

Received: 21 March 2016 Accepted: 7 September 2016

Published online: 20 September 2016

\section{References}

1. Endler JA. Geographic variation, speciation and clines. Princeton, New Jersey: Princeton University Press; 1977.

2. Nosil P. Ecological speciation. New York: Oxford University Press; 2012.

3. Schluter D. The ecology of adaptive radiation. New York: Oxford University Press; 2000

4. Lenormand T. From local adaptation to speciation: specialization and reinforcement. Int J Ecol. 2012;2012(Article ID 508458):1-11.

5. Slatkin M. Gene flow and the geographic structure of natural populations. Science. 1987;236(4803):787-92.

6. Smadja CM, Butlin RK. A framework for comparing processes of speciation in the presence of gene flow. Mol Ecol. 2011;20(24):5123-40.

7. Harrison RG, Larson EL. Hybridization, introgression, and the nature of species boundaries. J Hered. 2014:105:795-809.

8. Hoekstra HE, Drumm KE, Nachman MW. Ecological genetics of adaptive color polymorphism in pocket mice: geographic variation in selected and neutral genes. Evolution. 2004;58(6):1329-41.

9. Muñoz MM, Crawford NG, McGreevy TJ, Messana NJ, Tarvin RD, Revell L, Zandvliet RM, Hopwood JM, Mock E, Schneider AL, et al. Divergence in coloration and ecological speciation in the Anolis marmoratus species complex. Mol Ecol. 2013;22(10):2668-82

10. Rosenblum EB, Harmon LJ. "Same same but different": replicated ecological speciation at White Sands. Evolution. 2011;65(4):946-60.

11. Thorpe RS, Surget-Groba Y, Johansson H. Genetic tests for ecological and allopatric speciation in anoles on an island archipelago. PLOS Genet. 2010;6(4):e1000929.

12. Vignieri SN, Larson JG, Hoekstra HE. The selective advantage of crypsis in mice. Evolution. 2010;64(7):2153-8.

13. Dice LR: Effectiveness of selection by owls of deer mice (Peromyscus maniculatus) which contrast in color with their background. Contributions of the Laboratory of Vertebrate Biology, University of Michigan 1947, 34:1-20

14. Kaufman DW. Adaptive coloration in Peromyscus polionotus: experimental selection by owls. J Mammal. 1974;55(2):271-83.

15. $\mathrm{Ng} J$, Landeen EL, Logsdon RM, Glor RE. Correlation between Anolis lizard dewlap phenotype and environmental variation indicates adaptive divergence of a signal important to sexual selection and species recognition. Evolution. 2013;67(2):573-82.

16. Schwartz A. Geographic variation in Anolis distichus Cope (Lacertilia, Iguanidae) in the Bahama Islands and Hispaniola. Bull Mus Comp Zool. 1968;137:255-309.

17. Glor RE, Laport RG. Are subspecies of Anolis lizards that differ in dewlap color and pattern also genetically distinct? A mitochondrial analysis. Mol Phylogen Evol. 2012;64(2):255-60.

18. Williams EE, Case SM. Interactions among members of the Anolis distichus complex in and near the Sierra de Baoruco, Dominican Republic. J Herpetol. 1986;20(4):535-46.

19. Geneva AJ, Hilton J, Noll S, Glor RE. Multilocus phylogenetic analyses of Hispaniolan and Bahamian trunk anoles (distichus species group). Mol Phylogen Evol. 2015:87:105-17.

20. $\mathrm{Ng}$ J, Glor RE. Genetic differentiation among populations of a Hispaniolan trunk anole that exhibit geographical variation in dewlap colour. Mol Ecol. 2011;20(20):4302-17

21. de Queiroz K. The general lineage concept of species, species criteria, and the process of speciation: a conceptual unification and terminological 
recommendations. In: Howard DJ, Berlocher SH, editors. Endless Forms: Species and Speciation. Oxford: Oxford University Press; 1998. p. 57-75.

22. de Queiroz K. The general lineage concept of species and the defining properties of the species category. In: Wilson RA, editor. Species: New Interdisciplinary Essays. Cambridge, Massachusetts: MIT Press; 1999. p. 49-89.

23. Castañeda MR, Kd Q. Phylogenetic relationships of the Dactyloa clade of Anolis lizards based on nuclear and mitochondrial DNA sequence data. Mol Phylogen Evol. 2011;61(3):784-800.

24. Glor RE, Losos JB, Larson A. Out of Cuba: overwater dispersal and speciation among lizards in the Anolis carolinensis subgroup. Mol Ecol. 2005; 14(8):2419-32.

25. Jackman TR, Larson A, Queiroz KD, Losos JB. Phylogenetic relationships and tempo of early diversification in Anolis lizards. Syst Biol. 1999;48(2):254-85.

26. Kolbe JJ, Glor RE, Schettino LRG, Lara AC, Larson A, Losos JB. Genetic variation increases during biological invasion by a Cuban lizard. Nature. 2004:431(7005):177-81.

27. Nicholson KE, Glor RE, Kolbe JJ, Larson A, Hedges SB, Losos JB. Mainland colonization by island lizards. J Biogeogr. 2005;32(6):929-38.

28. Rabosky DL, Glor RE. Equilibrium speciation dynamics in a model adaptive radiation of island lizards. Proc Natl Acad Sci U S A. 2010;107(51):22178-83.

29. Wang IJ, Glor RE, Losos JB. Quantifying the roles of ecology and geography in spatial genetic divergence. Ecol Lett. 2013;16(2):175-82.

30. Brandley MC, De Queiroz K. Phylogeny, ecomorphological evolution, and historical biogeography of the Anolis cristatellus series. Herpetol Monogr. 2004;18:90-126.

31. Macey JR, Larson A, Ananjeva NB, Papenfuss TJ. Evolutionary shifts in three major structural features of the mitochondrial genome among iguanian lizards. J Mol Evol. 1997:44(6):660-74.

32. Glor RE, Gifford ME, Larson A, Losos JB, Schettino LR, Lara ARC, Jackman TR. Partial island submergence and speciation in an adaptive radiation: a multilocus analysis of the Cuban green anoles. Proc R Soc Lond Ser B Biol Sci. 2004:271(1554):2257-65.

33. Drummond AJ, Kearse M, Heled J, Moir R, Thierer T, Ashton B, Wilson A, StonesHavas S. Geneious v4.6.1. 2006. Available from http://www.geneious.com/.

34. Maddison DR, Maddison WP. MacClade 4: Analysis of phylogeny and character evolution. Version 4.0. Sunderland, Massachusetts: Sinauer Associates; 2000.

35. Kumazawa $Y$, Nishida M. Sequence evolution of mitochondrial transfer-RNA genes and deep-branch animal phylogenetics. J Mol Evol. 1993;37(4):380-98.

36. Stamatakis A. RAxML-VI-HPC: Maximum likelihood-based phylogenetic analyses with thousands of taxa and mixed models. Bioinformatics. 2006; 22(21):2688-90.

37. Huelsenbeck JP, Ronquist F. MRBAYES: Bayesian inference of phylogeny. Bioinformatics. 2001;17:754-5.

38. Ronquist F, Huelsenbeck JP. MRBAYES 3: Bayesian phylogenetic inference under mixed models. Bioinformatics. 2003;19:1572-4.

39. Lanfear R, Calcott B, Ho SYW, Guindon S. PartitionFinder: combined selection of partitioning schemes and substitution models for phylogenetic analyses. Mol Biol Evol. 2012;29(6):1695-701.

40. Sullivan J, Swofford D, Naylor G. The effect of taxon sampling on estimating rate heterogeneity parameters of maximum-likelihood models. Mol Biol Evol. 1999;16(10):1347-56.

41. Rambaut A, Suchard MA, Xie D, Drummond AJ. Tracer v1.6. Available from http://beast.bio.ed.ac.uk/Tracer. Accessed June 2016.

42. Clement M, Snell Q, Walke P, Posada D, Crandall K. TCS: estimating gene genealogies, Proc 16th Int Parallel Distrib Process Symp, vol. 2. 2002. p. 184.

43. Ng J, Perkins SL, Dussmann EJ, Glor RE. Eleven highly polymorphic microsatellite markers for the lizard Anolis distichus. Conserv Genet Resour. 2010;1(1):135-9.

44. Raymond M, Rousset F. GENEPOP (version 1.2): population genetics software for exact tests and ecumenicism. J Hered. 1995:86:248-9.

45. Rice WR. Analyzing tables of statistical tests. Evolution. 1989;43(1):223-5.

46. Gompert Z, Buerkle CA. A powerful regression-based method for admixture mapping of isolation across the genome of hybrids. Mol Ecol. 2009;18(6):1207-24.

47. Szymura JM, Barton NH. Genetic analysis of a hybrid zone between the fire-bellied toads, Bombina bombina and B. variegata, near Cracow in southern Poland. Evolution. 1986;40(6):1141-59.

48. Bowcock AM, Ruizlinares A, Tomfohrde J, Minch E, Kidd JR, Cavallisforza LL. High resolution of human evolutionary trees with polymorphic microsatellites. Nature. 1994;368(6470):455-7.
49. Dieringer D, Schlotterer C. MICROSATELLITE ANALYSER (MSA): a platform independent analysis tool for large microsatellite data sets. Mol Ecol Notes. 2003;3(1):167-9.

50. Murphy MA, Evans JS, Storfer A. Quantifying Bufo boreas connectivity in Yellowstone National Park with landscape genetics. Ecology. 2010;91(1):252-61.

51. Storfer A, Murphy MA, Spear SF, Holderegger R, Waits LP. Landscape genetics: where are we now? Mol Ecol. 2010;19(17):3496-514.

52. Pritchard JK, Stephens M, Donnelly P. Inference of population structure using multilocus genotype data. Genetics. 2000;155(2):945-59.

53. Falush D, Stephens M, Pritchard JK. Inference of population structure using multilocus genotype data: Linked loci and correlated allele frequencies. Genetics. 2003;164(4):1567-87.

54. Evanno G, Regnaut S, Goudet J. Detecting the number of clusters of individuals using the software STRUCTURE: a simulation study. Mol Ecol. 2005:14(8):2611-20.

55. Earl DA, vonHoldt BM. STRUCTURE HARVESTER: a website and program for visualizing STRUCTURE output and implementing the Evanno method. Conserv Genet Resour. 2012;4(2):359-61.

56. Coulon A, Fitzpatrick JW, Bowman R, Stith BM, Makarewich CA, Stenzler LM, Lovette IJ. Congruent population structure inferred from dispersal behaviour and intensive genetic surveys of the threatened Florida scrub-jay (Aphelocoma coerulescens). Mol Ecol. 2008;17(7):1685-701.

57. Berner D, Grandchamp A-C, Hendry AP. Variable progress toward ecological speciation in parapatry: stickleback across eight lake-stream transitions. Evolution. 2009;63(7):1740-53.

58. Saint-Laurent $R$, Legault M, Bernatchez L. Divergent selection maintains adaptive differentiation despite high gene flow between sympatric rainbow smelt ecotypes (Osmerus mordax Mitchill). Mol Ecol. 2003;12(2):315-30.

59. Schneider CJ, Losos JB, de Queiroz K. Evolutionary relationships of the Anolis bimaculatus group from the northern Lesser Antilles. J Herpetol. 2001; 35(1):1-12.

60. Smith TB, Schneider CJ, Holder K. Refugial isolation versus ecological gradients. Genetica. 2001;112-113(1):383-98.

61. Mullen LM, Hoekstra HE. Natural selection along an environmental gradient: A classic cline in mouse pigmentation. Evolution. 2008;62(7):1555-69.

62. Thorpe RS, Surget-Groba Y, Johansson H. Quantitative traits and mode of speciation in Martinique anoles. Mol Ecol. 2012;21(21):5299-308.

63. Funk DJ, Nosil P, Etges WJ. Ecological divergence exhibits consistently positive associations with reproductive isolation across disparate taxa. Proc Natl Acad Sci U S A. 2006;103(9):3209-13.

64. Rundle HD, Nosil P. Ecological speciation. Ecol Lett. 2005;8(3):336-52.

65. Schemske DW. Adaptation and the origin of species. Am Nat. 2010;176:S4-S25.

66. Servedio MR, Doorn GSV, Kopp M, Frame AM, Nosil P. Magic traits in speciation: 'magic' but not rare? Trends Ecol Evol. 2011;26(8):389-97.

67. Malhotra A, Thorpe RS. The dynamics of natural selection and vicariance in the Dominican anole: Patterns of within-island molecular and morphological divergence. Evolution. 2000;54(1):245-58.

68. Stenson AG, Malhotra A, Thorpe RS. Population differentiation and nuclear gene flow in the Dominican anole (Anolis oculatus). Mol Ecol. 2002; 11(9):1679-88.

69. Thorpe RS, Stenson AG. Phylogeny, paraphyly and ecological adaptation of the colour and pattern in the Anolis roquet complex on Martinique. Mol Ecol. 2003;12(1):117-32.

70. Grant C. Report on a collection of Hispaniolan reptiles. Herpetologica. 1956;12(2):85-90.

71. Lenormand T. Gene flow and the limits to natural selection. Trends Ecol Evol. 2002;17(4):183-9.

72. Nosil P, Harmon LJ, Seehausen O. Ecological explanations for (incomplete) speciation. Trends Ecol Evol. 2009;24(3):145-56.

73. Gavrilets S. Fitness landscapes and the origin of species. Princeton, NJ: Princeton University Press; 2004

74. Calsbeek R, Knouft JH, Smith TB. Variation in scale numbers is consistent with ecologically based natural selection acting within and between lizard species. Evol Ecol. 2006;20(4):377-94.

75. Malhotra A, Thorpe RS. Microgeographic variation in scalation of Anolis oculatus (Dominica, West Indies): a multivariate analysis. Herpetologica. 1997;53(1):49-62.

76. Nosil P, Funk DJ, Ortiz-Barrientos D. Divergent selection and heterogeneous genomic divergence. Mol Ecol. 2009;18(3):375-402.

77. Losos JB, Schluter D. Analysis of an evolutionary species-area relationship. Nature. 2000;408(6814):847-50. 